\title{
Bacterial Adhesion to Worn Silicone Hydrogel Contact Lenses
}

\author{
LÍVIA SANTOS, MSc, DIANA RODRIGUES, BSc, MADALENA LIRA, MSc, \\ M. ELISABETE C. D. REAL OLIVEIRA, PhD, ROSARIO OLIVEIRA, PhD, \\ EVA YEBRA-PIMENTEL VILAR, $\mathrm{PhD}$, and JOANA AZEREDO, $\mathrm{PhD}$
}

\author{
Centro de Engenharia Biológica, Universidade do Minho, Portugal (LS, DR, RO, JA), Centro de Física, Universidade do Minho, Portugal \\ (ML, MECDRO), and Departamento de Optica e Optometria, Universidade de Santiago de Compostela, Spain (EY-PV)
}

\begin{abstract}
Purpose. The aim of this study was to, firstly, investigate whether silicone-hydrogel contact lenses (CL) are more or less susceptible to bacterial adhesion than conventional ones and, secondly, assess the influence of lens wear in the extent of bacterial adhesion. Four silicone-hydrogel CL (galyfilcon A, balafilcon A, lotrafilcon A, and lotrafilcon B) and one conventional hydrogel (etafilcon A) CL were tested.

Methods. Bacterial adhesion experiments were performed on unworn and worn CL using the strain Staphylococcus epidermidis 9142 . Worn lenses were obtained from a group of 31 subjects fitted with a silicone-hydrogel CL in one eye and a conventional hydrogel $\mathrm{CL}$ as contralateral pair. These lenses were used on a daily basis in combination with a multipurpose lens care solution. Adhesion assays were carried out in a parallel plate flow chamber, followed by image analysis. Hydrophobicity, roughness, and topography of the lenses surfaces were assessed through contact angle measurements and atomic force microscopy.

Results. Unworn conventional and silicone-hydrogel CL were equally susceptible to bacterial adhesion of $S$. epidermidis. Conversely, worn conventional hydrogel (etafilcon A) were more prone to bacterial adhesion than worn silicone-hydrogel materials, which exhibited similar adhesion extents among them. The results also showed that the lens surface properties such as hydrophobicity, roughness, and surface topography changed during wear. The alteration of surface hydrophobicity of silicone and conventional hydrogel CL during wear had a great impact on lens bacterial adhesion susceptibility. Accordingly, balafilcon A becomes significantly less hydrophobic and less prone to bacterial adhesion after lens wear, whereas etafilcon A becomes more hydrophobic and also more susceptible to bacterial adhesion $(p<0.05)$.

Conclusions. Worn silicone-hydrogel galyfilcon A, balafilcon A, lotrafilcon A, and lotrafilcon B are equally prone to microbial adhesion of $S$. epidermidis and generally less susceptible than the conventional hydrogel.
\end{abstract}

(Optom Vis Sci 2008;85:520-525)

Key Words: silicone hydrogel, AFM, S. epidermidis adhesion

M icrobial keratitis is a rare but serious ocular infection which can lead to permanent vision loss. Extended wear, ${ }^{1}$ ocular trauma, ${ }^{2,3}$ hypoxia ${ }^{3}$ and lack of compliance, ${ }^{3}$ among other factors, are predisposing issues for its occurrence.

With the introduction of silicone-hydrogel contact lenses (CL) several improvements were achieved. Overnight edema was found to be similar to those found on non-CL wearers and much smaller than observed on conventional hydrogel CL wearers as a result of their high oxygen transmissibility. ${ }^{4}$ It was also found that the incidence of microbial keratitis was five times smaller than with conventional hydrogel lenses, for extended wearing periods. ${ }^{5}$ This lower incidence seems to be associated with their higher oxygen transmissibility. However, some authors have also suggested that it could be related to less bacterial binding to the lens surface as well. ${ }^{6}$

The first aim of this study was to verify whether silicone-hydrogel CLs bind fewer bacteria than hydroxyethylmethacrylate (HEMA)based conventional ones, which might serve to reduce the incidence of microbial keratitis. The second aim involved assessing the influence of lens wear in the extent of adhesion. For this purpose, in vitro microbial adhesion assays onto four unworn and worn silicone-hydrogel CL, and one conventional hydrogel CL were performed. It is well established that an increase in surface hydro- 
TABLE 1.

Contact lenses properties

\begin{tabular}{|c|c|c|c|c|c|}
\hline Commercial name & Manufacturer & Material & FDA group & Water content (\%) & Surface treatment \\
\hline Acuvue & $\begin{array}{l}\text { Johnson \& Johnson } \\
\text { Vision Care }\end{array}$ & Etafilcon A & IV & 58 & No \\
\hline Acuvue Advance & $\begin{array}{l}\text { Johnson \& Johnson } \\
\text { Vision Care }\end{array}$ & Galyfilcon & 1 & 47 & No \\
\hline Purevision & Bausch \& Lomb & Balafilcon A & III & 36 & Plasma oxidation \\
\hline Focus Night \& Day & CIBA Vision & Lotrafilcon A & 1 & 24 & $\begin{array}{l}25 \mathrm{~nm} \text { plasma coating with } \\
\text { high refractive index }\end{array}$ \\
\hline $\mathrm{O}_{2}$ Optix & CIBA Vision & Lotrafilcon B & 1 & 33 & $\begin{array}{l}25 \mathrm{~nm} \text { plasma coating with } \\
\text { high refractive index }\end{array}$ \\
\hline
\end{tabular}

phobicity or roughness can lead to an increase of bacterial adhesion susceptibility. ${ }^{7,8}$ Therefore, these properties were also assessed in this work. Microbial adhesion studies to silicone-hydrogel have been previously performed by several authors. ${ }^{9-11}$ However, the present work covers a wider range of CL materials with different surface treatments, some of them never evaluated before with respect to roughness and bacterial adhesion. ${ }^{9-11}$

\section{MATERIALS AND METHODS Contact Lenses}

The properties of the CLs used in this study are detailed in Table 1. Four silicone (galyfilcon A, balafilcon A, lotrafilcon A, and lotrafilcon B) and one conventional hydrogel (etafilcon A) CL were tested in their unworn and worn states. The silicone-hydrogel lenses are surface treated by gas plasma during manufacturing, except galyfilcon that has an incorporated wetting agent (Hydraclear).

\section{Experimental Design}

Thirty-one subjects from both genders were enrolled in this study, excluding any lost to follow-up. The average age of the subjects was $23.6 \pm 5.5$ years and they were chosen according to the following parameters: they had never worn CL before (neophytes), they were not taking any medications during the trial, they did not suffer from any kind of ocular allergy, and they had no tendency for dry eye syndrome.

The subjects were randomly divided into four groups. Each group wore a specific type of silicone-hydrogel lens and a conventional one as contralateral pair in a single masked fashion way. The silicone-hydrogel CL were monthly replaced and the conventional hydrogel every 15 days, for 6 months. Apart from physiological changes, no complications occurred during the trial. For the adhesion studies 8 galyfilcon $\mathrm{A}$, balafilcon $\mathrm{A}$, lotrafilcon $\mathrm{A}$ and etafilcon $A$, and 7 lotrafilcon B CL were assayed. CL were worn on a daily wear schedule of between 12 and 14 hours. The subjects were instructed to remove the lenses at the end of this period and to soak them overnight in a multipurpose solution (ReNu MultiPlus, Bausch and Lomb, polyhexanide $0.0001 \%$, hydranate $0.03 \%$, and poloxamine $1 \%$ ).

At the end of the wearing period, each lens was aseptically removed from the eye and placed in a sterile vial containing a sterile saline solution $(0.9 \% \mathrm{NaCl})$. Vials were labeled with a code and details of the lens material. The CL were stored at $4^{\circ} \mathrm{C}$ for no longer than 5 days until analysis.

The research followed the tenets of the Declaration of Helsinki. Informed consent was obtained from the subjects after explanation of the nature and possible consequences of this study.

According to the manufacturer's recommendation, galyfilcon A and lotrafilcon B should be replaced every two weeks. However, in this study they were worn for 30 days to establish a comparison with the other lenses which are recommended for use up to 30 days. It must be stressed that no complications resulted from the extension of the wearing period.

\section{Bacterial Strain and Growth Conditions}

The bacterium S. epidermidis 9142 was used in the present study. This species is a Gram-positive pathogen commonly involved in extended wear microbial keratitis ${ }^{12}$ and was therefore chosen for the in vitro adhesion assays. S. epidermidis 9142 is a well-known producer of the polysaccharide that promotes coagulase negative staphylococci adherence and biofilm formation, which is referred to as either polysaccharide intercellular adhesin or by its chemical composition, poly- $\mathrm{N}$-acetyl glucosamine. This strain was kindly provided by Dr. Gerald B. Pier, Harvard Medical School, Boston, U.S. and its adhesion and biofilm formation capabilities have been characterized in previous studies. ${ }^{13,14}$

A $4^{\circ} \mathrm{C}$ culture stock was inoculated into an Erlenmeyer flask containing $10 \mathrm{ml}$ of tryptic soy broth (Merck, Germany) and incubated at $37^{\circ} \mathrm{C}$ for 24 hours. Following this period, $1 \mathrm{ml}$ of the culture suspension was transferred to a second Erlenmeyer flask containing $30 \mathrm{ml}$ of tryptic soy broth and incubated at $37^{\circ} \mathrm{C}$ for 18 hours to obtain a mid-exponential growth culture. Cells were harvested by centrifugation ( 15 minutes, $4000 \mathrm{rpm}$ ) and washed twice with ultrapure water and finally, the cells were resuspended in phosphate buffer saline (PBS, $8 \mathrm{~g} \mathrm{l}^{-1} \mathrm{NaCl}, 0.2 \mathrm{~g} \mathrm{l}^{-1} \mathrm{KCl}, 0.2 \mathrm{~g} \mathrm{l}^{-1}$ $\mathrm{KH}_{2} \mathrm{PO}_{4}, 1.15 \mathrm{~g} \mathrm{l}^{-1} \mathrm{Na}_{2} \mathrm{HPO}_{4}, \mathrm{pH} 7.4$ ).

\section{Adhesion Assays and Image Analysis}

The adhesion assays were performed with unworn and worn CL in a parallel plate flow chamber. Two opposite edges of each CL were cut to flatten the surface and the lens mounted on the bottom plate of the flow chamber. All the tubes and the flow chamber were filled with a PBS solution (special care was taken to remove all air 
bubbles from the system) which circulated through the system for 15 minutes. A pulse-free flow was established by hydrostatic pressure, and the suspension was fed using a peristaltic roller pump. Afterwards, the flow was switched to a bacterial suspension $(6 \times$ $10^{10} \mathrm{CFU} / \mathrm{ml}$ ) that circulated throughout the system at room temperature for 120 minutes in laminar regime, at a flow rate of $2 \mathrm{E}-4$ $\mathrm{ml} / \mathrm{s}$. After this period, fresh PBS was circulated throughout the system to remove the non-adhered or loosely adhered cells. Cell quantification was performed using a phase contrast microscope (Carl Zeiss, Germany) connected to a charge-coupled device video camera (Carl Zeiss, Germany) that acquires images at a magnification of $1622 \times$ with a resolution of $1300 \times 1030$ pixels. Twenty images were randomly taken from each lens. To eliminate image interference, the background was captured and subtracted from the original image. Cells were entmmerated using the Sigma Scan Pro program and, with the magnification used, $1 \mathrm{~cm}^{2}$ was equivalent to 5110 captured images. These measurements were repeated seven or eight times for each lens material.

\section{Hydrophobicity}

CL hydrophobicity has been assessed in other studies. Some authors $^{15}$ have estimated lens surface hydrophobicity using the sessile drop technique whereas, more recently, other authors ${ }^{16}$ have made use of the advancing type technique. In the present study, water contact angle measurements were performed with Millipore ultrapure water using the advancing type technique and an apparatus OCA 20 (Dataphysics, Germany). Each lens were cut into four pieces and placed on a microscope slide. The excess moisture was removed by gentle blotting with absorbent paper. The measurements were carried out on $4 \mathrm{CL}$ from each type and repeated four times on each lens piece.

\section{Roughness}

Surface roughness was assessed through atomic force microscopy (AFM) using the Tapping Mode (PicoScan Controller, Molecular Imaging, U.S.). Measurements were performed in a liquid cell containing $\mathrm{PBS}$ and using a $\mathrm{V}$-shaped $\mathrm{Si}_{3} \mathrm{~N}_{4}$ cantilever with a constant spring of $0.58 \mathrm{~N} / \mathrm{m}$. AFM assays were performed on unworn and worn CL. Roughness was expressed as average roughness (Ra) of the surface.

The analyzed lenses were balafiloon $A$, lotrafilcon $B$, and gallyfilcon A. Balafilcon A and lotrafilcon B exhibit different types of surface treatment and galyfilcon A incorporates wetting agents as an alternative to surface treatment. The analyzed area for balafilcon A was $100 \mu \mathrm{m}^{2}$ due to the fact that some surface details can only be observed with this frame, ${ }^{17}$ whereas galyfilcon $\mathrm{A}$ and lotrafilcon $\mathrm{B}$ were analyzed within a $25 \mu \mathrm{m}^{2}$ frame. The measurements were repeated three or four times per CL material.

\section{Data Analysis}

Hydrophobicity data were evaluated by the one-way analysis of variance test. The extent of bacterial adhesion and lens roughness was statistically evaluated through the non-parametric MannWhitney $\mathrm{U}$ test because the data were not normally distributed. All tests were performed with a $95 \%$ confidence level using the statis- tical program SPSS (Statistical Package for the Social Sciences). Two distinct comparisons were made: between lens materials and within the same material before and after lens wear.

\section{RESULTS \\ Bacterial Adhesion of Staphylococcus epidermidis}

The number of adhered cell to unworn and worn CL is presented in Fig. 1. No statistical differences were found among unworn CL ( $p>$

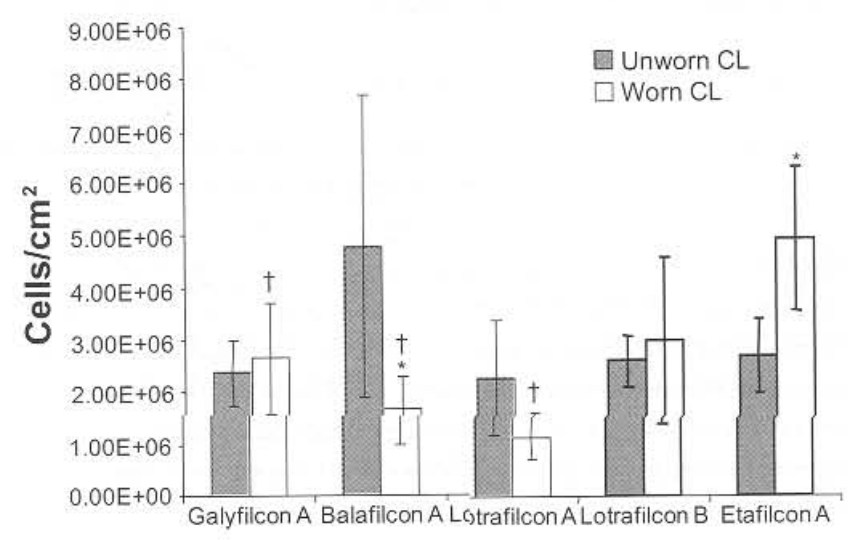

\section{FIGURE 1.}

Number of adhered cells to unworn and worn CL. *Statistically different compared to unworn CL. tStatistically different from worn etafilcon A. Error bars represent standard deviations $(n=7)$ (Mann-Whitney U Test).

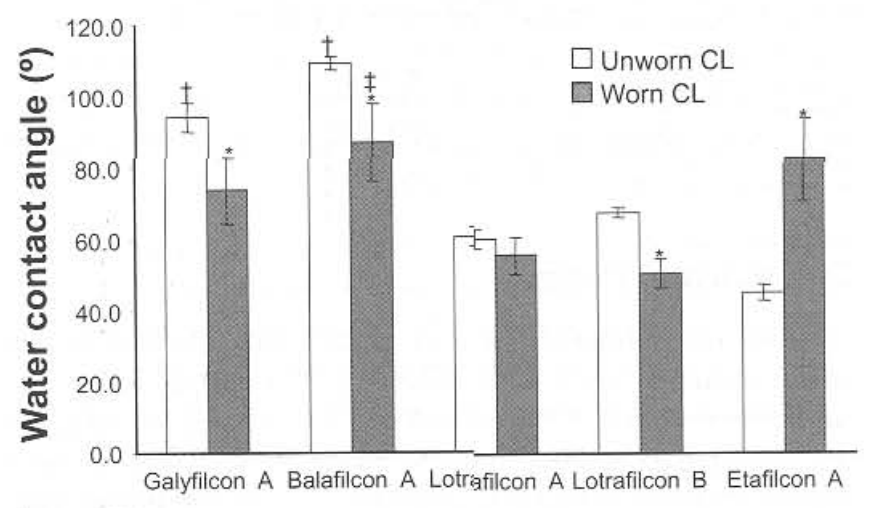

\section{FIGURE 2.}

Contact angle (advancing) measurements performed with water to unworn and worn $\mathrm{CL}$. *Statistically different from unworn lenses. +Statistically different from fotrafilcon $A$, lotrafilicon $B$ and unworn etafilcon $A$. FStatistically different from worn galyfilcon $\mathrm{A}$, lotrafilcon $\mathrm{A}$, and lotrafilcon $\mathrm{B}$. Error bars represent standard deviations $(n=16)$ (One-way ANOVA performed with $95 \%$ of confidence level).

\section{TABLE 2.}

Mean roughness of unworn and worn $\mathrm{CL}(\mathrm{nm})$

\begin{tabular}{lccc}
\hline & $\begin{array}{c}\text { Galyfilcon A } \\
(5 \times 5 \mu \mathrm{m})\end{array}$ & $\begin{array}{c}\text { Balafilcon A } \\
(10 \times 10 \mu \mathrm{m})\end{array}$ & $\begin{array}{c}\text { Lotrafilcon B } \\
(5 \times 5 \mu \mathrm{m})\end{array}$ \\
\hline Unworn & $2.32 \pm 0.085^{\mathrm{a}}$ & $7.04 \pm 0.66$ & $4.51 \pm 2.83$ \\
Worn & $30.09 \pm 11.27^{\mathrm{b}, \mathrm{c}}$ & $17.63 \pm 14.78^{\mathrm{b}}$ & $4.96 \pm 4.12$ \\
\hline
\end{tabular}

(Mann-whitney with 95\% confidence level) $(n=3)$.

aStatistically different from unworn balafilcon A and lotrafilcon B.

bStatistically different from unworn CL.

"Statistically different from worn lotrafilcon B. 


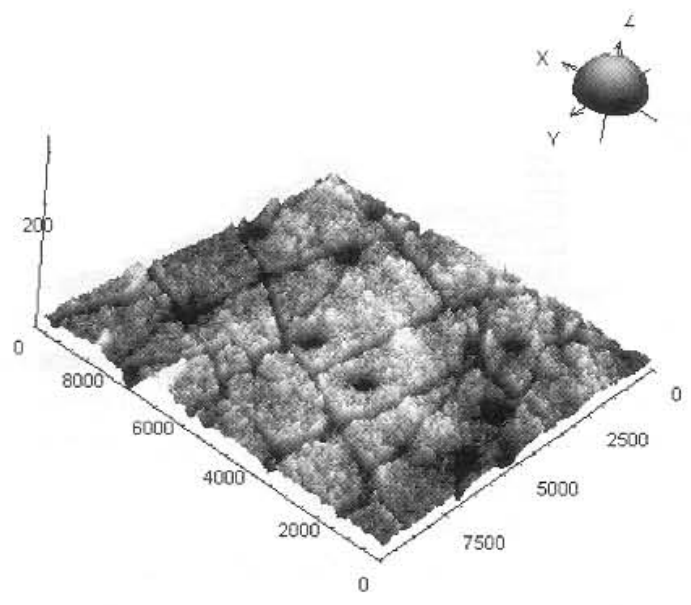

a) Unworn

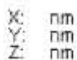

FIGURE 3.

Topography of balafilcon A before (a) and after (b) wear.

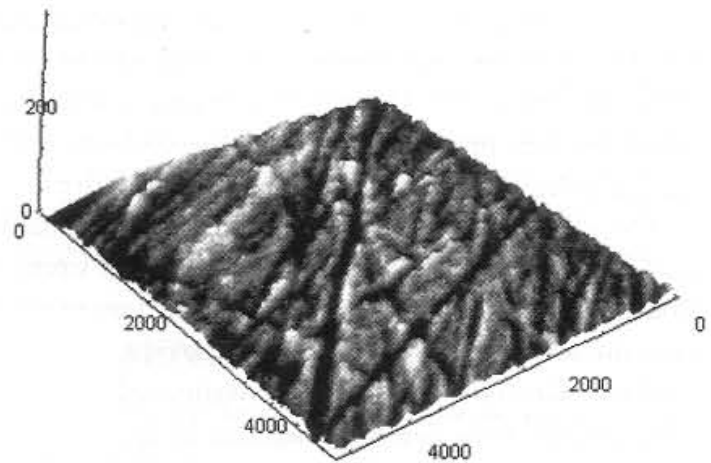

a) Unworn

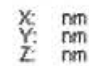

FIGURE 4.

Topography of lotrafilcon B before (a) and after (b) wear.

0.05). Comparing worn CL, silicone-hydrogel materials showed to be less prone to bacterial adhesion than the conventional hydrogel lens $(\mathrm{p}<0.05)$. Lotrafilcon $B$ was the only exception, exhibiting a value $(\mathrm{p}=0.055)$ that is almost statistically significant.

After wear, balafilcon A became less susceptible to bacterial adhesion, but no statistical differences were found among the other silicone-hydrogel materials. Conversely, the conventional hydrogel became more prone to bacterial adhesion.

\section{Hydrophobicity}

Inspection of Fig. 2 reveals that unworn silicone-hydrogel CL have water contact angles $>50^{\circ}$ (the breakpoint between hydrophilicity and hydrophobicity) and are relatively more hydrophobic than the conventional hydrogel. Balafilcon $\mathrm{A}$ and galyfilcon showed to be the most hydrophobic ones $(p<0.05)$. After lens wear, silicone-hydrogel CL became less hydrophobic $(p<0.05)$ and balafilcon A remained relatively more hydrophobic than the
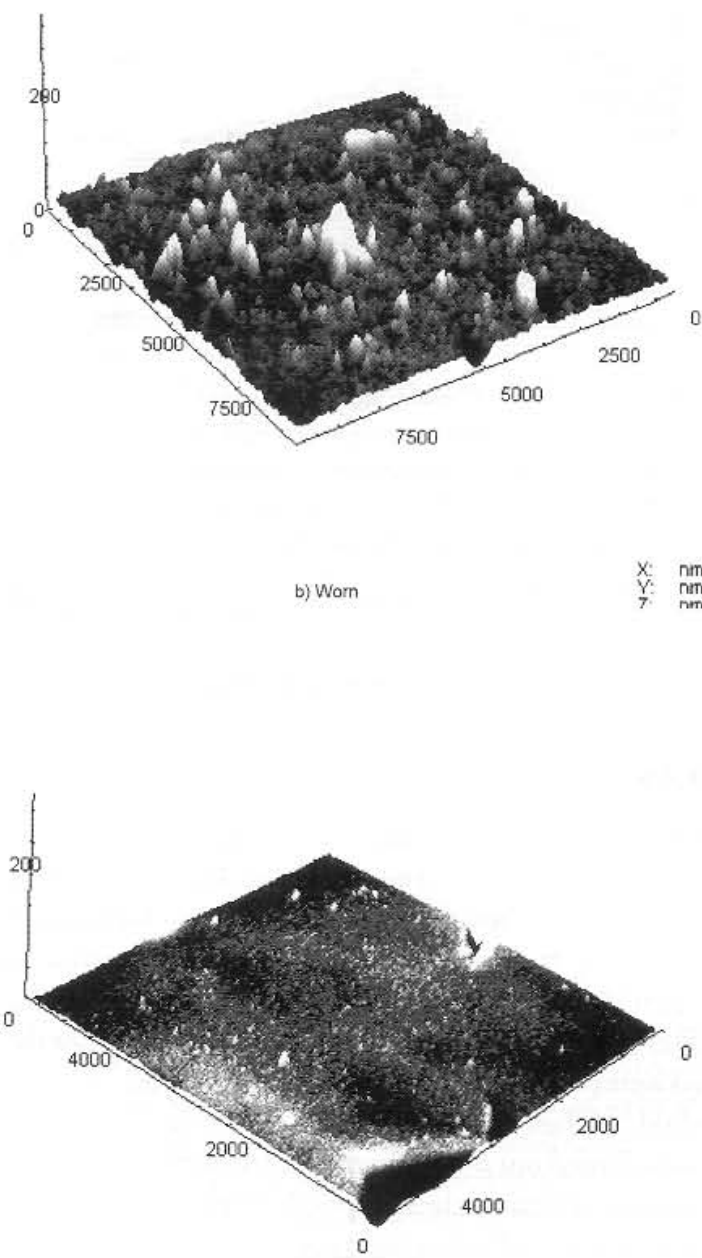

b) Worn $\begin{array}{ll}\mathrm{X} & \mathrm{nm} \\ \mathrm{Y} & \mathrm{nm} \\ \mathrm{Z}: & \mathrm{nm}\end{array}$

other materials $(\mathrm{p}<0.05)$. Conversely, the conventional hydrogel etafilcon A, after being worn, displayed an increase in the water contact angle values from $44.8^{\circ} \pm 2.4$ to $82.3^{\circ} \pm 11.6$.

\section{Roughness and Topography}

The mean roughness $\left(R_{a}\right)$ values are detailed in Table 2 . Analysis of unworn CL revealed that galyfilcon A seems to exhibit the smoothest surface $(p=0.05)$. After lens wear, galyfilcon $A$ and balafilcon A roughness increased and galyfilcon A became significantly rougher than lotrafilcon $B(p=0.05)$.

As can be observed in Figs. 3 and 4, balafilcon A shows the presence of macropores, whereas some grooves can be seen in lotrafilcon B, respectively. These structures disappeared after wear in both cases. Fig. 5 confirms that galyfilcon $\mathrm{A}$ has a very smooth and uniform surface. Galyfilcon A, despite becoming rougher after lens wear, did maintain the same smooth appearance. 


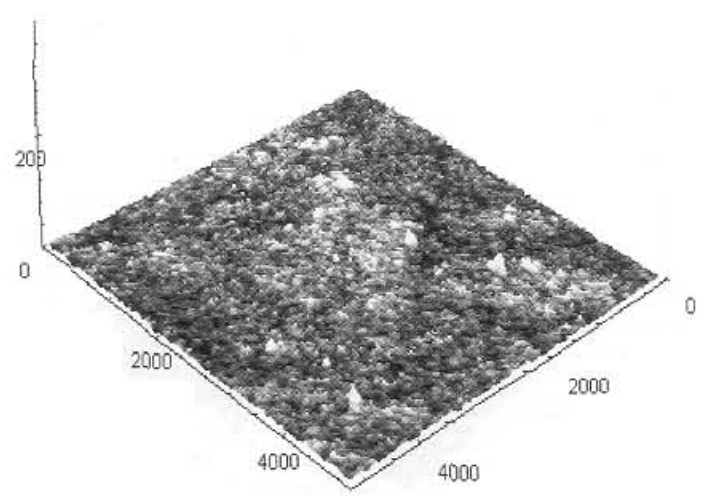

a) Unworn

FIGURE 5 .

Topography of galyfilcon A before (a) and after (b) wear.

\section{DISCUSSION}

The present study focuses on bacterial adhesion to four siliconehydrogel CLs and one conventional hydrogel lens. The main purpose was to compare the propensity of silicone-hydrogel CIs for bacterial adhesion and to verify if these materials are more or less prone to bacterial adhesion than a conventional hydrogel material. A similar comparative study was previously performed; however, in that case the adhesion assays were performed only on unworn CL and using a static adhesion method. ${ }^{17}$ Therefore, in this work the in vitro adherence experiments were carried out on both worn and unworn materials to address the influence of wear on the susceptibility for adhesion. Moreover, in the present work, adhesion was assessed by a dynamic adhesion method, using a parallel plate flow chamber as this is a more realistic methodology and virtually induces less variability. ${ }^{18}$

The results obtained in the present work revealed that worn silicone-hydrogel CL exhibited similar levels of adhered bacteria and are thus equally prone to microbial colonization. However, when compared with worn etafilcon A, these materials generally bind fewer cells (Fig. 1). This fact supports the hypothesis that silicone-hydrogels potentially induce fewer cases of microbial keratitis on account of their weaker susceptibility to $S$. epidermidis binding, although this is only true for worn CL. In fact, the susceptibility for adhesion of unworn silicone and conventional hydrogels is the same (Fig. 1) or even higher for silicone-hydrogel, as reported in a previous study. ${ }^{17}$ However, it shall be considered that as soon as a CL is placed in the eye it becomes rapidly conditioned by the tear film molecules that greatly influence bacterial colonization.

This study demonstrates that wear has a great impact on CL adhesion with special relevance for balafilcon $\mathrm{A}$ and etafilcon $\mathrm{A}$ adhesion susceptibilities. In fact, the extent of S. epidermidis adhesion to the silicone-hydrogel decreased when worn. This evidence corroborates the results of previous studies, in which $P$. aeruginosa adhered in a slightly lower degree (not statistically significant) to worn balafilcon $A$ ? and $P$. aeruginosa adhered in a lesser extent to worn lotrafilcon $A$ and balafilcon A materials. ${ }^{10}$ The fact that worn conventional hydrogel is more prone to $S$. epidermidis adhesion than unworn is contradicted by a previous study in which worn etafilcon $\mathrm{A}$ lenses became less suscep-

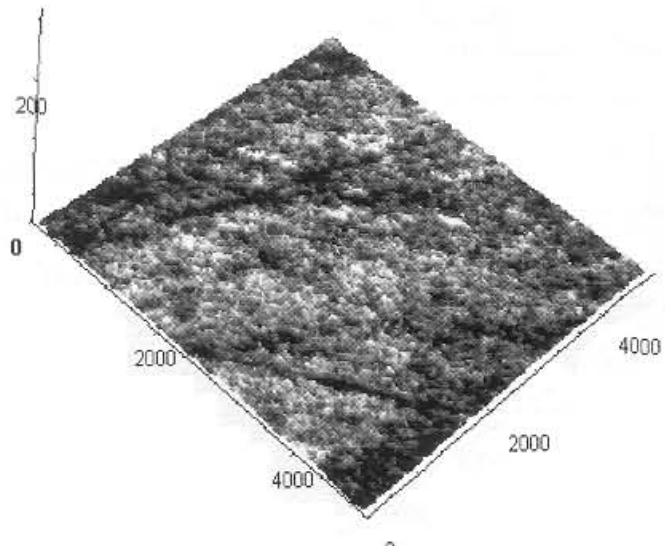

0

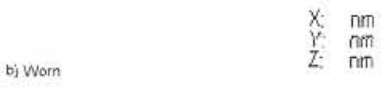

$\begin{array}{lll}X & n m \\ Y & n \pi m \\ Z: & n \pi m\end{array}$ tible to $P$. aeruginosa adhesion. ${ }^{19}$ Some authors have suggested that CL wear increases susceptibility to bacterial adhesion whereas others show the reverse. ${ }^{9-11}$ The use of different strains, methods, wear schedules, lens materials, maintenance solutions, and subjects are certainly the underlying reasons for this disparity.

The use of only one strain and one conventional hydrogel CL could be considered a limitation of our work since, and as mentioned before, the strain involved and the lens material can influence the adhesion extent. Therefore, the results obtained could not hold true for all bacteria and all conventional hydrogels. However, it has already been demonstrated that $S$. epidermidis is one of the most frequent pathogens colonizing $\mathrm{CL}^{10,19}$ and, together with $P$. aeruginosa, is one of the most common bacteria involved in microbial keratitis. ${ }^{15}$ Also, etafilcon $\mathrm{A}$ is one of the most popular conventional hydrogel $\mathrm{CL}$ and thus it can be considered representative to establish a comparison with silicone-hydrogel materials.

It is generally recognized that silicone-hydrogel CLs are relatively more hydrophobic than conventional hydrogel lenses ${ }^{15}$ (Fig. 2) and therefore potentially bind more microbes. ${ }^{\top}$ Previous reports also found that balafilcon $A$ and galyfilcon $A$ have the most hydrophobic surfaces. ${ }^{16,20}$ However, this study reveals that their hydrophobicity significantly decreases after wear, which may explain their lower adhesion capability. The adsorption of proteins and lipids from the tear film may have induced these changes, since these molecules are amphiphilic and are therefore capable of modifying the surface hydrophobicity. For silicone-hydrogels, the orientation of the hydrophilic regions of adsorbed molecules to the outer environment may render the lens surface less hydrophobic. Conversely, the conventional hydrogel etafilcon A, which is hydrophilic, must have adsorbed the proteins and lipids through their hydrophilic region thus orienting their hydrophobic region to the outer environment. As the surface becomes less hydrophilic, it attracts more lipids which in turn increase surface hydrophobicity. ${ }^{21}$

It is generally accepted that roughness may boost microbial adhesion, but that was not observed in this study. In fact, although surface roughness has increased in most of the worn lens, the adherence capability did not follow the same tendency. It is possible 
that the decrease of hydrophobicity might have neutralized the roughness impact. Additionally, surface topography may have played an important role in this process. For example, in the case of balafilcon, despite the increase in roughness of this worn lens, the surface crevices and macroporous disappeared after wear and this may have contributed to a decrease in adherence. Still, AFM analysis was a useful tool to study the impact of wear on lens roughness and topography. This aspect is discussed elsewhere and related with lens clinical performances. ${ }^{22}$ Nonetheless, in this article we would like to stress the increase in roughness of galyfilcon $A$ and balafilcon A after lens wear. Because these lenses possess polyvinyl pyrrolidone, a liposoluble monomer, it is possible that lipids from the tear film are responsible for such augmentation. However, an in vitro study assessing lipid deposition (data not shown) revealed that lipid deposition on galyfilcon A was not greater than that observed for other silicone-hydrogel lenses. The total quantity of adsorbed proteins onto this worn lens is similar to that observed in other silicone-hydrogel lenses, ${ }^{20}$ which also excludes the hypothesis of higher protein adsorption. Therefore, we address the possibility that polyvinyl pyrrolidone could have been lost during wear, resulting in such increment in roughness.

In summary, this study demonstrates that worn silicone-hydrogel galyfilcon A, balafilcon A, lotrafilcon A, and lotrafilcon $\mathrm{B}$ are equally prone to microbial colonization and generally less susceptible than the conventional hydrogel, suggesting that wearing this type of material does not increase the risk of developing ocular events associated to $S$. epidermidis colonization.

\section{ACKNOWLEDGMENTS}

This work was supported by the Portuguese Foundation for Science and Technology (FCT) through the project POCTI/FCB/44,628/2002 and also from the grant $B D 19,679 / 2004$.

Received August 21, 2007; accepted February 13, 2008.

\section{REFERENCES}

1. Willcox MD, Holden BA. Contact lens related corneal infections. Biosci Rep 2001;21:445-61.

2. Keay L, Edwards K, Naduvilath T, Taylor HR, Snibson GR, Forde K, Stapleton F. Microbial keratitis predisposing factors and morbidity. Ophthalmology 2006;113:109-16.

3. Weissman BA, Mondino BJ. Risk factors for contact lens associated microbial keratitis. Cont Lens Anterior Eye 2002;25:3-9.

4. Jones L, Dumbleton K. Silicone-hydrogel contact lenses. Part 1. Evolution and current status. Optom Today 2002;20:26-32. Available at: http://www.optometry.co.uk/articles/docs/ 2a85c209e250a4b2b829811247ea977d_jones20020920.pdf. Accessed on March 27, 2008.

5. Morgan PB, Efron N, Hill EA, Raynor MK, Whiting MA, Tullo AB. Incidence of keratitis of varying severity among contact lens wearers. Br J Ophthalmol 2005;89:430-6.

6. Cavanagh HD, Ladage PM, Li SL, Yamamoto K, Molai M, Ren DH, Petroll WM, Jester JV. Effects of daily and overnight wear of a novel hyper oxygen-transmissible soft contact lens on bacterial binding and corneal epithelium: a 13-month clinical trial. Ophthalmology 2002;109: $1957-69$.

7. Bos R, van der Mei HC, Busscher HJ. Physico-chemistry of initial microbial adhesive interactions-its mechanisms and methods for study. FEMS Microbiol Rev 1999;23:179-230.
8. Packham DE. Surface energy, topography and adhesion. Int J Adhesion Adhesives 2003;23:437-48.

9. Borazjani RN, Levy B, Ahearn DG. Relative primary adhesion of Pseudomonas aeruginosa, Serratia marcescens and Staphylococcus aureus to HEMA-type contact lenses and an extended wear silicone hydrogel contact lens of high oxygen permeability. Cont Lens Anterior Eye 2004;27: 3-8.

10. Vermeltfoort PB, Rustema-Abbing M, de Vries J, Bruinsma GM, Busscher HJ, van der Linden ML, Hooymans JM, van der Mei HC. Influence of day and night wear on surface properties of silicone hydrogel contact lenses and bacterial adhesion. Cornea 2006;25: 516-23.

11. Willcox MD, Harmis N, Cowell, Williams T, Holden. Bacterial interactions with contact lenses; effects of lens material, lens wear and microbial physiology. Biomaterials 2001;22:3235-47.

12. Gristina AG, Naylor PT. Implant-associated infection. In: Ratner BD, Hoffman AS, Schoen FJ, Lemons JE, eds. Biomaterials Science: An Introduction to Materials in Medicine. San Diego: Academic Press; 1996:205-14.

13. Cerca N, Pier GB, Vilanova M, Oliveira R, Azeredo J. Quantitative analysis of adhesion and biofilm formation on hydrophilic and hydrophobic surfaces of clinical isolates of Staphylococcus epidermidis. Res Microbiol 2005;156:506-14.

14. Cerca N, Jefferson KK, Maira-Litran T, Pier DB, Kelly-Quintos C, Goldmann DA, Azeredo J, Pier GB. Molecular basis for preferential protective efficacy of antibodies directed to the poorly acetylated form of staphylococcal poly-N-acetyl-beta-(1-6)-glucosamine. Infect Immun 2007;75:3406-13.

15. Jones L, Senchyna M, Glasier MA, Schickler J, Forbes I, Louie D, May C. Lysozyme and lipid deposition on silicone hydrogel contact lens materials. Eye Contact Lens 2003;29(suppl):S75-9.

16. Maldonado-Codina C, Morgan PB. In vitro water wettability of silicone hydrogel contact lenses determined using the sessile drop and captive bubble techniques. J Biomed Mater Res A 2007;83:496-502.

17. Henriques M, Sousa C, Lira M, Elisabete M, Oliveira R, Azeredo J. Adhesion of Pseudomonas aeruginosa and Staphylococcus epidermidis to silicone-hydrogel contact lenses. Optom Vis Sci 2005;82:446-50.

18. Cerca N, Pier GB, Oliveira R, Azeredo J. Comparative evaluation of coagulase-negative staphylococci (CoNS) adherence to acrylic by a static method and a parallel-plate flow dynamic method. Res Microbiol 2004;155:755-60.

19. Cheng L, Muller SJ, Radke CJ. Wettability of silicone-hydrogel contact lenses in the presence of tear-film components. Curr Eye Res 2004;28:93-108.

20. Santos L, Rodrigues D, Lira M, Oliveira ME, Oliveira R, Vilar EY, Azeredo J. The influence of surface treatment on hydrophobicity, protein adsorption and microbial colonisation of silicone hydrogel contact lenses. Cont Lens Anterior Eye 2007;30:183-8.

21. Bontempo AR, Rapp J. Protein-lipid interaction on the surface of a hydrophilic contact lens in vitro. Curr Eye Res 1997;16:776-81.

22. Lira M, Santos L, Azeredo J, Yebra-Pimentel E, Oliveira ME. Comparative study of silicone-hydrogel contact lenses surfaces before and after wear using atomic force microscopy. J Biomed Mater Res B Appl Biomater 2008;85:361-7.

Joana Azeredo
IBB-Institute for Biotechnology and Bioengineering
Centre for Biological Engineering
Universidade do Minho
Campus de Gualtar
$4710-057$ Braga, Portugal
e-mail: jazeredo@deb.uminho.pt

